\title{
COMPARACIÓN CLÍNICA Y RADIOGRÁFICA DE PULPOTOMÍAS REALIZADAS CON ELECTROCAUTERIO Y FORMOCRESOL. EN MOLARES DECIDUOS: ESTUDIO PRELIMINAR
}

\author{
${ }^{1}$ Beatriz Duarte P., ${ }^{1}$ Adriana Medina P., ${ }^{1}$ Laura Liliana Pérez R., ${ }^{2}$ Martha Juliana Rodríguez G. \\ ${ }^{1}$ Estudiante X semestre, Facultad de Odontología, U. Santo Tomás, ${ }^{2}$ Odontóloga U. Javeriana, \\ Odontopediatra C.E.S., Docente U. Santo Tomás.
}

Autor responsable de correspondencia: Dra. Martha Juliana Rodríguez $G$.

Correo electrónico: marthajuro@yahoo.com

\begin{abstract}
RESUMEN
Objetivo: Comparar clínica y radiográficamente las pulpotomías realizadas con electrocauterio y con formocresol en molares deciduos, en niños de 3 a 9 años.

Materiales y métodos: La muestra estuvo conformada por diecisiete individuos. En total se trataron 28 molares distribuidos aleatoriamente de la siguiente manera: Trece en el Grupo 1 (electrocauterio) y quince al Grupo 2 (formocresol). Todos los molares se trataron con aislamiento absoluto. Después de la remoción de la caries, se retiró el techo de la cámara pulpar. Luego de lograr la hemostasia, se procedió a colocar la punta activa No. 4 del electrocauterio sobre los muñones pulpares durante cinco segundos en el Grupo 1, mientras que en el Grupo 2 se colocó una torunda de algodón impregnada con formocresol durante cinco minutos. Posteriormente, en ambos grupos se colocó una base de óxido de zinc - eugenol y se hizo la restauración con corona de acero. Se realizó el seguimiento de lo dientes por un periodo de 8 a 30 días.

Resultados: Se obtuvo éxito clínico (ausencia de movilidad y fístula) del 100\% en ambos grupos. Con relación a los hallazgos radiográficos, se obtuvo un 100\% de éxito en el Grupo 1; en el Grupo 2, el éxito disminuyó al 83.4\%.

Conclusiones: Los resultados no fueron estadísticamente significativos. Sin embargo, se observó que el electrocauterio podría ser una buena alternativa para realizar pulpotomías en los molares primarios. [Duarte B, Medina A, Pérez LL, Rodríguez M]. Comparación clínica y radiográfica de pulpotomías realizadas con electrocauterio y Formocresol en molares deciduos: Estudio preliminar. Ustasalud Odontología 2004; 3: 48 - 54]
\end{abstract}

Palabras clave: Electrocauterio, Formocresol, Pulpotomía.

\section{CLINICAL AND RADIOGRAPHICAL COMPARISON OF PULPOTOMY TECHNIQUE WITH ELECTROSURGERY AND FORMOCRESOL. IN PRIMARY MOL.ARS: PRELIMINAR REPORT}

\begin{abstract}
Purpose: The aim of this study was to compare the effect of electrosurgery to that of formocresol as pulp dressing agents in pulpotomized primary molars with carious pulp exposure.

Material and methods: Twenty-eight primary molars of 17 children were treated pulpotomy technique. The teeth were randomly assigned to the electrosurgery (group 1, experimental) and formocresol (group 2, control). All teeth were treated with rubber dam. After removing tooth decay, pulp chamber was reached. The pulp stumps were touched with the electrode in mode three in the group 1. In the group 2, formocresol was placed with a cotton pellet over the pulp stumps for 5 minutes and removed; the pulp stumps were then covered by zinc oxide - eugenol paste. The teeth of both groups were restored with stainless steel crowns. The follow - up evaluation ranged from 8 to 30 days.

Results: A clinical success (absence of mobility and fistulae) was obtained in 100\% of both groups. Radiographically, 100\% success was obtained in group one while in group the success decreased to $83.4 \%$.

Conclusions: The results were not statistically significant. However, the electrosurgery could be a good alternative for pulpotomy technique in primary molars.
\end{abstract}

Key words: Electrosurgery, Formocresol, Pulpotomy. 


\section{INTRODUCCIÓN}

La pulpotomia se define como la remoción quirúrgica de la porción coronal de la pulpa. Este procedimiento está basado en el principio que la pulpa radicular se encuentra sana y es capaz de cicatrizar después de la amputación del tejido pulpar cameral infectado y/0 afectado. Su objetivo es preservar la vitalidad de la pulpa radicular manteniendo el órgano dental libre de signos y/o síntomas adversos para el paciente. ${ }^{1}$

Se considera una de las técnicas de terapia pulpar más utilizada en la consulta odontológica pediátrica; se enseña y se aplica en el $98 \%$ de los departamentos de Odontopediatría en los Estados Unidos. ${ }^{2}$

El tejido pulpar que se encuentra en los conductos radiculares es tratado con un agente, farmacológico o no farmacológico, que promueve la regeneración, la desvitalización o la preservación del tejido pulpar remanente. ${ }^{3}$

El formocresol es uno de los medicamentos más utilizados para la realización de pulpotomías en molares deciduos porque ha demostrado un éxito clínico y radiográfico hasta de un $96 \%{ }^{46}$ Sin embargo, su éxito histológico es debatible. ${ }^{7,8}$ Se acepta que causa una desvitalización del tejido pulpar, aunque las investigaciones difieren de la extensión de tal efecto. ${ }^{9}$ También, existe controversia en torno al uso de este material por su efecto tóxico y su absorción sistémica. ${ }^{10-12}$

Por estas razones han aparecido nuevas alternativas para la realización de pulpotomías en dientes deciduos. ${ }^{13-16}$ Entre éstas se encuentra el electrocauterio. ${ }^{17,18}$

La electrocirugía se conoce desde que G. S. Wyeth desarrolló el generador de alta frecuencia en $1924 .{ }^{18}$ Este es un medio físico que emplea una fuente de calor sin producir toxicidad alguna para el organismo ya que no es un método farmacológico y no existe distribución sistémica. ${ }^{18,20-21}$

Sin embargo, los estudios sobre el uso del electrocauterio en pulpotomías de molares deciduos no han sido concluyentes. Los resultados obtenidos han causado discrepancias. $^{20-21}$
El propósito del presente estudio preliminar es comparar clínica y radiográficamente pulpotomías realizadas con formocresol y con electrocauterio, en molares deciduos, para determinar las diferencias entre ambos.

\section{MATERIALES Y MÉTODOS}

Se realizó un ensayo clínico controlado, simultáneo, con enmascaramiento doble para comparar los efectos clínicos y radiográficos de las pulpotomías realizadas con electrocauterio y formocresol en molares deciduos.

La población del estudio estuvo conformada por infantes, con edades entre 3 y 9 años, de ambos géneros, procedentes del área metropolitana de Bucaramanga en el período comprendido entre septiembre de 2001 y mayo de 2002, cuyos padres aceptaron voluntariamente su participación en el estudio, firmando la Carta de Consentimiento Informado.

Dentro de los criterios de inclusión se tuvieron en cuenta los primeros y/o segundos molares asintomáticos, que presentaran exposición pulpar por caries y que pudieran ser restaurados con coronas de acero. Se excluyeron del estudio los molares sintomáticos, con fístula o edema, con radiolucidez periapical o de furca o con presencia de hemorragia abundante, después de la amputación de la pulpa cameral.

La muestra fue de conveniencia, estuvo conformada por 17 individuos que cumplieron con los criterios de inclusión. Considerando que fue un estudio simultáneo, cada individuo debía cumplir, el criterio de dos molares primarios que requirieran pulpotomía, uno por cada grupo (Grupo 1: Electrocauterio, y Grupo 2: Formocresol). En el caso de que un individuo presentara tres molares primarios para la técnica de pulpotomía, fue aleatoriamente asignado a cualquiera de los dos grupos de tratamiento.

Se analizó la edad y el género. Dentro de la valoración odontológica se analizó el dolor, la presencia de fístula, la reabsorción interna y externa, y la movilidad. El dolor se midió por medio de la escala Escala de Dolor Facial (FPS), la cual indica la sensación de satisfacción o molestia en niños de tres a nueve años. ${ }^{24}$ Está conformada por un cartón que contiene nueve caras, que presentan diferentes expresiones faciales; éstas van desde la sonrisa a las lágrimas (Figura 1). 
Se realizó doble enmascaramiento para evitar sesgo; dos personas realizaron la intervención. El paciente no conoció cómo se asignó el tratamiento a cada molar, ya sea con electrocauterio o con formocresol.

\section{Procedimiento}

GRUPO 1: Electrocauterio.

- Toma de radiografía previa.

- Aplicación del anestésico local (Roxicaina ${ }^{\circledR}$ al 2\%, con vasoconstrictor).

- Aislamiento del campo con dique de goma (Figura 2).

- Remoción de la lesión cariosa con pieza de alta velocidad refrigerada. Se usó una fresa redonda No. 4 de diamante.

- Remoción del techo de la cámara pulpar.

- Remoción de la pulpa cameral con cucharilla Maillefer No. 5 (Figura 3).

- Control de la hemorragia con torundas de algodón estériles (Figura 4).

- Colocación de la punta activa No. 4 del electrocauterio (Parkell Sensimatic 500 SE) sobre los muñones pulpares durante cinco segundos ${ }^{25}$ (Figuras 5 y 6 ).

- Colocación de la base de óxido de zinc - eugenol.

- Toma de radiografía.

- Restauración con corona de acero (Figura 7 y 8).

El equipo de electrocauterio fue usado en el Modo 3 Coagulación que es más efectivo, preciso y genera una destrucción mínima de tejido. ${ }^{25}$ La técnica con el electrocauterio fue usada, en todos los casos, por un solo investigador.

En algunos casos fue necesario realizar una aplicación adicional con la punta activa del electrocauterio debido a hemorragia posterior a la aplicación inicial. Si esto ocurría, se esperaban diez segundos entre la primera aplicación y la segunda. ${ }^{18}$

GRUPO 2: Formocresol.

- Toma de radiografía previa.

- Aplicación del anestésico local (Roxicaina ${ }^{\circledR}$ al 2\%, con vasoconstrictor).

- Aislamiento del campo con dique de goma.

- Remoción de la lesión cariosa con pieza de alta velocidad refrigerada. Se usó una fresa redonda No. 4 de diamante.

- Remoción del techo de la cámara pulpar.

- Remoción de la pulpa cameral con cucharilla

Maillefer No. 5.

- Control de la hemorragia con torundas de algodón estériles.
- Colocación de una torunda de algodón impregnada con Formocresol (Eufar ${ }^{\circledR}$ ) durante cinco minutos.

- Colocación de la base de óxido de zinc - eugenol.

- Toma de radiografía.

- Restauración con corona de acero.

\section{Análisis estadístico}

Se realizó aplicando un test exacto, de Fischer, con un nivel de significancia alpha $=0.05(\mathrm{p}=0.26)$. Al analizar el poder del estudio se determinó del 10\%, lo que implica un poder muy bajo y sugiere la necesidad de incrementar el tamaño de la muestra para concluir que no hay diferencia significativa entre los dos procedimientos. El análisis y la base de datos se elaboró en Epi - Info $6.02 .^{26}$

\section{Criterios éticos}

La realización de esta investigación siguió las recomendaciones para investigación biomédica de la Declaración de Helsinki, de la Asociación Médica Mundial, y las normas científico - técnicas y administrativas para la investigación en salud, Resolución No. 008430, de 1993, emanada del Ministerio de Salud, Título II, Capítulo 1, sobre Aspectos Éticos de la Investigación en Humanos, Artículos 5 al 11.

El proyecto fue presentado al Comité de Ética de la Universidad Santo Tomás, que dió su aprobación.

\section{RESULTADOS}

Se trataron un total de diecisiete pacientes en los que se realizaron 28 pulpotomías (13 con electrocauterio y 15 con formocresol), a partir de septiembre de 2001 hasta mayo de 2002. La presentación de los dientes de acuerdo con el tipo de molar y el material usado se encuentra en la Tabla 1.

\begin{tabular}{|c|c|c|c|}
\hline DIENTES & ELECTROC. & FORMOCRESOL & TOTAL \\
\hline \multicolumn{4}{|c|}{ Maxilar Superior } \\
\hline 1er. molar & 4 & 2 & 6 \\
\hline 2do. molar & 2 & 0 & 2 \\
\hline \multicolumn{4}{|c|}{ Maxilar Inferior } \\
\hline 1er. molar & 5 & 9 & 14 \\
\hline 2do. molar & 2 & 4 & 6 \\
\hline TOTAL. & 13 & 15 & 28 \\
\hline
\end{tabular}

Tabla 1. Distribución por localización de los molares tratados 


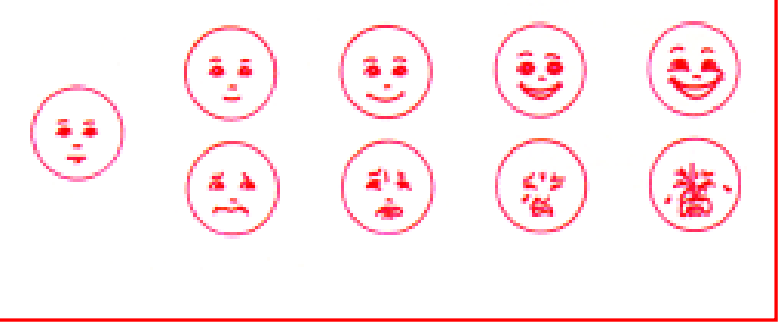

Figura 1. Escala de dolor facial. ${ }^{24}$

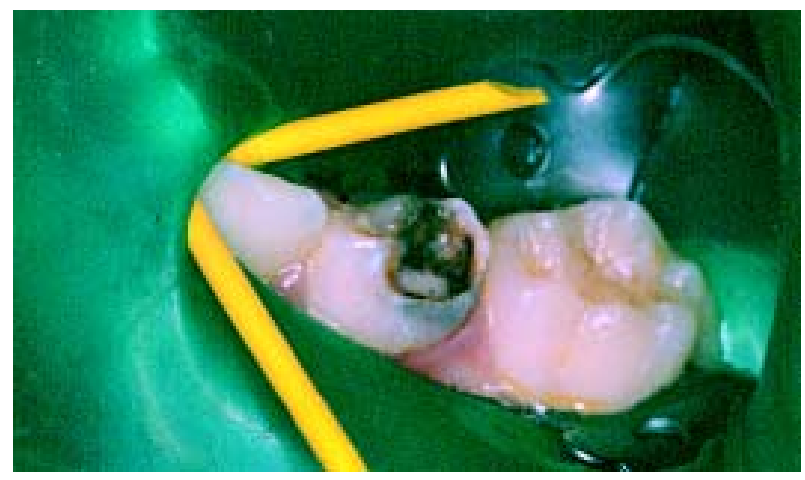

Figura 2. Primer molar inferior izquierdo.

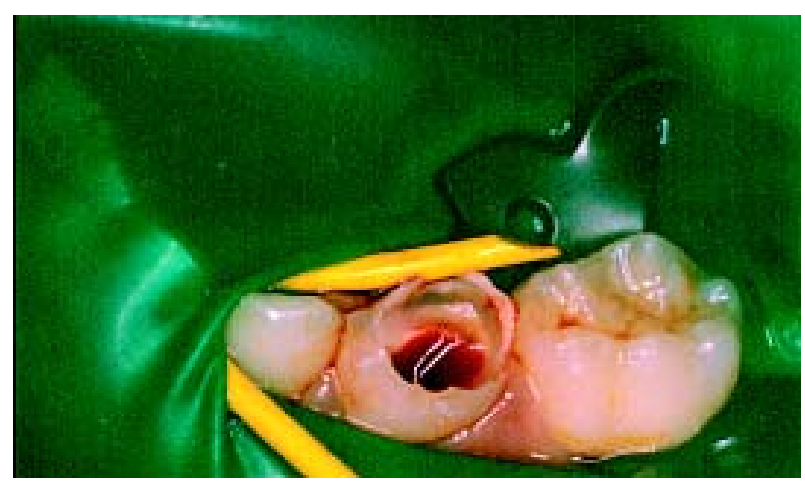

Figura 3. Exposición de la cámara pulpar.

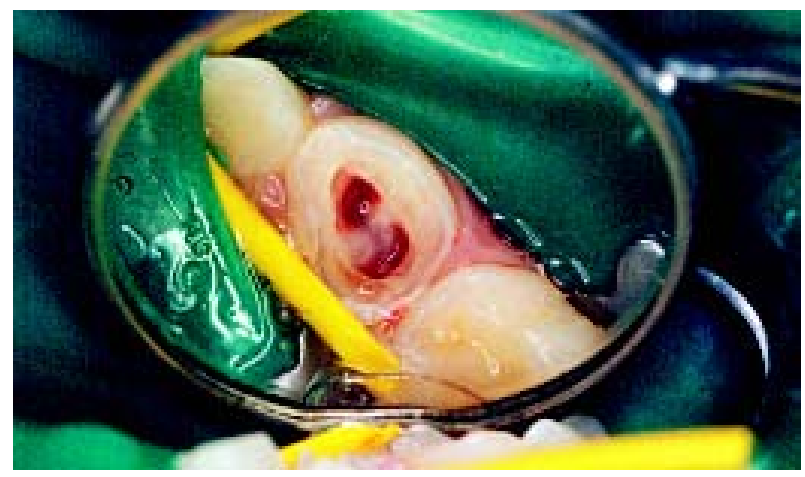

Figura 4. Control de la hemorragía con torundas de algodón.

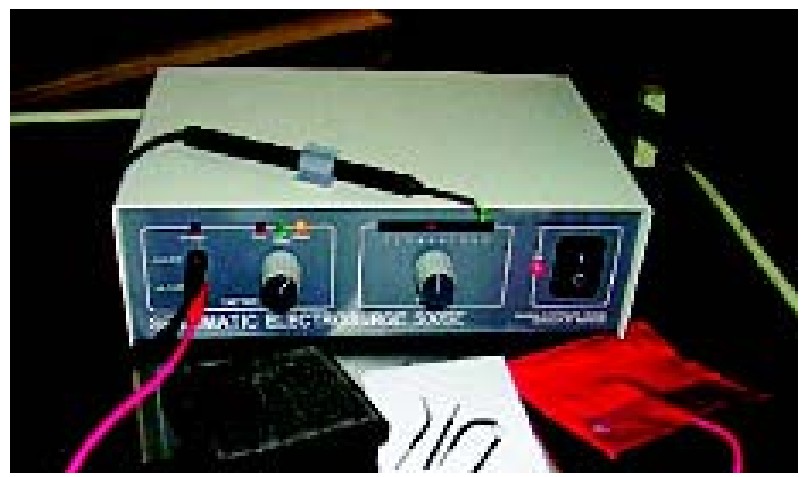

Figura 5. Electrocauterio. ${ }^{25}$

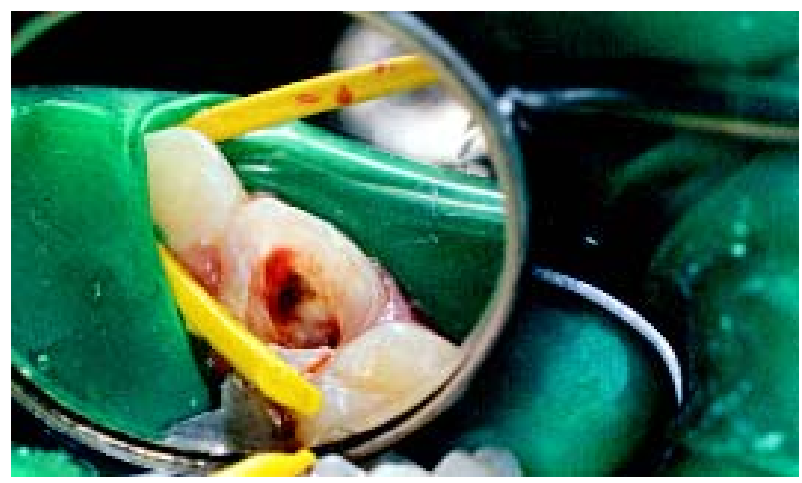

Figura 6. Apariencia cameral luego de la aplicación del electrocauterio.

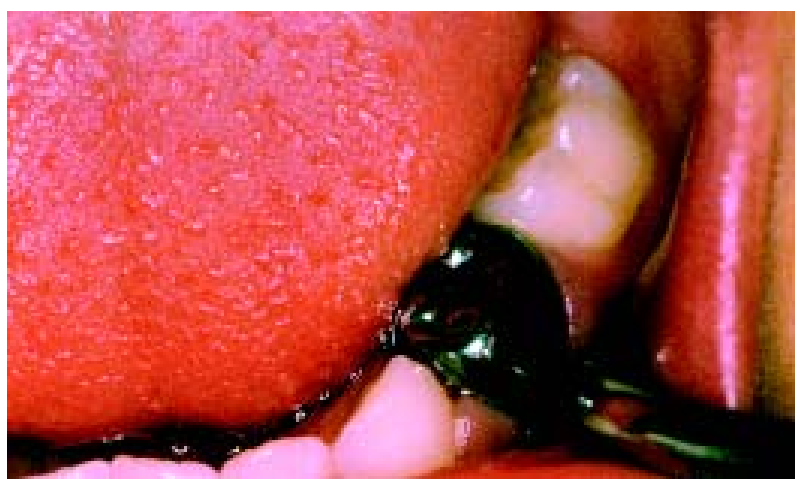

Figura 7. Cementación de una corona de acero.

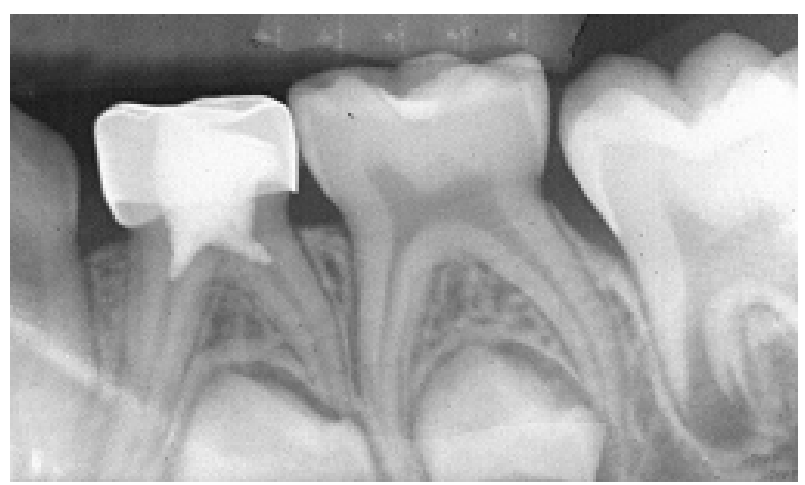

Figura 8. Radiografía final. 
En algunos procedimientos se realizaron controles, hasta por siete meses; sin embargo, fue necesario estandarizar el tiempo de todos los procedimientos a treinta días para poder evaluar los efectos de los dos agentes y compararlos (Tabla 2).

\begin{tabular}{lccccc}
\hline & \multicolumn{5}{c}{ Tiempo de seguimiento (meses) } \\
\hline & $<1$ & $1-2$ & $2 \cdot 3$ & $3 \cdot 4$ & $>4$ \\
Electrocauterio & 1 & 5 & 4 & 1 & 2 \\
Formocresol & 5 & 2 & 2 & 0 & 6 \\
Total & 6 & 7 & 6 & 1 & 8 \\
\hline
\end{tabular}

Tabla 2. Máximo periodo de seguimiento de los molares con pulpotomias.

\section{Evaluación clínica}

Para la evaluación clínica se excluyó un molar de un paciente, que reportó dolor previo al procedimiento con electrocauterio, quedando un total de 12 pulpotomías con electrocauterio y 15 con formocresol. Clínicamente, se evaluaron ausencia de movilidad y fistula, durante los ocho, quince y treinta días de haber realizado el procedimiento.

Todos los pacientes asistieron a los controles y se determinó un éxito clínico del $100 \%$ en el grupo del electrocauterio y del $100 \%$ en el grupo del formocresol (Tabla 3).

\section{Evaluación radiográfica}

Se examinaron un total de veintiocho (28) dientes distribuidos así: Trece (13) molares tenían tratamiento con electrocauterio y quince (15) con formocresol. Para la evaluación, se excluyeron dos molares tratados con electrocauterio y tres molares con formocresol, debido a deficiencias en la técnica radiográfica y en el proceso de revelado; además, uno de los molares tratados con electrocauterio presentó reabsorción externa en la radiografía inicial (Tabla 4).

\begin{tabular}{lcc}
\hline Técnica Empleada & $\boldsymbol{n}$ & $\%$ \\
\hline Electrocauterio & 11 & 47.8 \\
Formocresol & 12 & 52.2 \\
Total & 23 & 100 \\
\hline
\end{tabular}

Tabla 4. Número de molares evaluados radiográficamente.

En el Grupo 1 no se encontraron hallazgos radiográficos patológicos en T1, T2 y T3. En el Grupo 2 se encontró un (1) molar con radiolucidez interradicular en T3 y un (1) molar con radiolucidez periapical también en T3 (Tabla 5).

Los hallazgos radiográficos patológicos encontrados en el Grupo 2 correspondieron a pacientes diferentes.

\section{Efectos colaterales}

Al evaluar el dolor con la SFP (Escala de Dolor Facial), no se experimentó dolor en ninguno de los individuos tratados durante el tiempo de seguimiento.

\section{DISCUSIÓN}

Entre las diferentes alternativas de tratamiento para realizar la pulpotomía $a^{12-16}$ se seleccionó el electrocauterio como objeto de estudio para ser comparado con el formocresol, ya que éste desarrolla un medio físico no tóxico; es una técnica rápida y eficaz; se ha demostrado un éxito clínico y radiográfico hasta del 99.4\%, al evaluar 164 molares en un período de 70 meses. ${ }^{18}$

\begin{tabular}{lccccccc}
\hline \multicolumn{1}{c}{ E.ECTROCAUTERIO } & \multicolumn{5}{c}{ FORMOCRESOL. } \\
\hline HALLAZGos & T 1 & T 2 & T 3 & T 1 & T 2 & T 3 & TOTA \\
\multicolumn{1}{c}{ c.ínICos } & $n$ & $n$ & $n$ & $n$ & $n$ & $n$ & $n$ \\
Movilidad & 0 & 0 & 0 & 0 & 0 & 0 & 0 \\
Fistula & 0 & 0 & 0 & 0 & 0 & 0 & 0 \\
TOTAL. & 0 & 0 & 0 & 0 & 0 & 0 & 0 \\
\hline T1 = 8 dias. T2 = 15 dias. T3 $=30$ dias. \\
Tabla 3. Hallazgos clinicos.
\end{tabular}




\begin{tabular}{lcccccc}
\hline & \multicolumn{3}{c}{ ElECTROCAUTERIO } & \multicolumn{3}{c}{ FORMOCRESOL } \\
\hline & T 1 & T 2 & T 3 & T 1 & T 2 & T 3 \\
& $n(\%)$ & $n(\%)$ & $n(\%)$ & $n(\%)$ & $n(\%)$ & $n(\%)$ \\
Reabsorción interna & 0 & 0 & 0 & 0 & 0 & 0 \\
Reabsorción externa & 0 & 0 & 0 & 0 & 0 & 0 \\
Radiolucidez interradicular & 0 & 0 & 0 & 0 & 0 & $1(2.15)$ \\
Radiolucidez periapical & 0 & 0 & 0 & 0 & 0 & $1(2.15)$ \\
TOTAL & 0 & 0 & 0 & 0 & 0 & $2(4.3)$ \\
\hline
\end{tabular}

$\mathrm{T} 1=8$ dias. $\mathrm{T} 2=15$ dias. $\mathrm{T} 3=30$ dias.

Tabla 5. Hallazgos radiográficos

El formocresol ha sido el medicamento tradicional para realizar pulpotomías en molares deciduos debido a su amplio margen de éxito clínico y radiográfico. ${ }^{25,6}$ Sin embargo, los resultados histológicos obtenidos en algunas investigaciones no apoyan del todo su uso, por haberse presentado diversidad de resultados; se ha demostrado la absorción sistémica del medicamento en modelos animales., ${ }^{5,10,11}$

Los molares se evaluaron en un rango de tiempo de treinta días, porque todos cumplían con ese criterio.

Los resultados obtenidos con el electrocauterio indicaron un éxito clínico del 100\%, lo que se compara con estudios previos. ${ }^{18,20}$ Estos estudios no muestran coincidencia en la onda de alta frecuencia aplicada sobre el muñón pulpar. En el estudio de Mack y Dean se usó la electofulguración, con un éxito clínico y radiográfico del $99.4 \% .{ }^{18}$ Mientras, Shelter y Morton usaron el corte, con un éxito clínico y radiográfico del $91 \%$, en 11 dientes. ${ }^{20}$

En este estudio se usó como onda de alta frecuencia la coagulación con una intensidad de calor de 5 , recomendada por el Manual de Instrucciones del aparato. ${ }^{25}$

El éxito clínico de los molares tratados con formocresol fue del 100\%, similar al obtenido por Redig en $1968 .{ }^{4}$ No fue así, para la evaluación radiográfica, en la que se encontraron dos casos de alteración patológica. La radiolucidez periapical e interradicular, observada en este Grupo se presentó en el T3, es decir, a los 30 días post -operatorios. En la evaluación radiográfica previa (T0) no se observaron tales hallazgos. Es dudoso observar que se presente este signo radiográfico en un período tan corto de tiempo. En estudios histológicos se ha observa- do que la pulpa inicia un proceso de inflamación, llegando algunas veces a la reabsorción interna o a la necrosis pulpar en períodos mayores de tiempo. ${ }^{3,27}$

Clínica y radiográficamente, estos molares cumplieron todos los criterios de inclusión. Los hallazgos radiográficos encontrados se pueden atribuir a:

- No se estandarizó la técnica en la toma de la radiografía y en el proceso de revelado.

- Los molares podían estar sufriendo cambios histopatológicos imposibles de observar radiográficamente.

Los resultados obtenidos en este estudio contradicen los argumentos sobre la "sensibilidad al diagnóstico" del formocresol. Sheller y Morton encontraron que la técnica con electrocauterio es menos sensible al diagnóstico, comparada con la técnica del formocresol. Es decir, esta última puede ser exitosa, aun si el diagnóstico no es el más acertado. ${ }^{20}$

Este estudio es considerado como prueba piloto por el pequeño número molares y el período de seguimiento relativamente corto. Los pacientes atendidos en este estudio correspondían a niños que no tenían un domicilio establecido, por lo cual fue difícil localizarlos en las fechas estipuladas para los controles clínicos y radiográficos.

Hasta que se puedan realizar estudios más extensos, sería prematuro recomendar este tratamiento para tejidos pulpares expuestos por caries en molares deciduos. 


\section{BIBLIOGRAFÍA}

1. American Academy of Pediatric Dentistry. Guidelines for Pulp Therapy for Primary and Young Permanet Teeth. Ped Dent Special Issue 2000; 22: $67-68$.

2. Primosch R, Glomb T, Jerrell R. Primary tooth pulp therapy as taught in predoctoral pediatric dental programs in the United States. Ped Dent 1997; 19: $118-122$.

3. Ranly D. Pulpotomy therapy in primary teeth: New modalities for old rationales. Ped Dent 1994; 16: 403 - 409.

4. Redig D. A comparison and evaluation of two formocresol pulpotomy technics utilizing "Buckley's" formocresol. J Dent Child 1968; 35: 22 - 29.

5. Rölling I, Thylstrup A. A 3 - year follow - up study of pulpotomized primary molars treated with the formocresol technique. Scand J Dent Res 1975; 47: 47 - 53.

6. Hicks JM, Barr ES, Flaitz CM. Formocresol pulpotomies in primary molars: A radiographic study in a pediatric dentistry practice. J Pedodontics 1986; 10: 331 - 339.

7. Fuks A, Bimstein E, Bruchim A. Radiographic and histologic evaluation of the effect of two concentrations of formocresol on pulpotomized primary and young permanent teeth in monkeys. Ped Dent 1983; 5: $9-13$.

8. Doyle W, Mitchell D, McDonald R. Formocresol versus calcium hydroxide in pulpotomy. J Dent Child 1962; 29: 86 - 97.

9. Ranly D, Lazzari E. The formocresol pulpotomy - The past, the present and the future. J Pedod 1978; 2: $115-127$.

10. Myers D, Sheaf K, Kirksen T, Pashley D, Withford G, Reynolds K. Distribution fo C - 14 formaldehyde after pulpotomy with formocresol. J Am Dent Assoc 1978; 96: 805 - 813 .

11. Pashley EL, Myers D, Pashley D, Withford G. Systemic distribution fo $\mathrm{C}-14$ formaldehyde from formocresol treated pulpotomy sites. J Dent Res 1980; 59: $603-608$.

12. Waterhouse PJ. Formocresol and alternative primary molar pulpotomy medicaments: A review. Endod Dent Traumatol 1995; 11: $157-162$.

13. Fuks A, Holan G, Davis J, Eidelman E. Ferric sulfate versus diluted formocresol in pulpotomiezed primary molars: Long term follow -up. Ped Dent 1997; 19: 327 - 330.

14. Liu J, Chen L, Chao S. Laser pulpotomy of primary teeth. Ped Dent 1999; 21: 128.

15. Eidelman E, Holan G, Fuks A. Mineral trioxide aggregate vs. formocresol in pulpotomized primary molars: a preliminary report. Ped Dent 2001; 23: 15 - 18.
16. Waterhouse PJ, Nunn J, Whitworth JM. An investigation of the relative efficacy of Buckley's Formocresol and calcium hydroxide in primary molar vital pulp therapy. Brith Dent J 2000; 188: $1-11$.

17. Fishman S, Udin R, Good D, Rodef F. Success of electrofulguration pulpotomies covered by zinc oxide and eugenol or calcium hydroxide: A clinical study. Ped Dent 1996; 18: 385 390.

18. Mack RB, Dean JA. Electrosurgical pulpotomy: A retrospective human study. J Dent Child 1993; 60: 107 - 114.

19. Anderman I. Pedodontic electrosurgery. J Pedodont 1989; 14 : $202-213$.

20. Sheller B, Morton TH. Electrosurgical pulpotomy: A pilot study in humans. J. Endod 1987; 13: $69-76$.

21. Shulman ER, McIver FT, Burkes EJ. Comparison of electrosurgery and formocresol as pulpotomy techniques in monkey primary teeth. Pediatr Dent 1987; 9: 189- 194.

22. Shaw DW, Sheller B, Barrus BD, Morton TH. Electrosurgical pulpotomy - a 6 month study in primates. J Endod 1987; 13: 500 $-505$.

23. El-Meligy O, Abdalla M, El-Baraway S, El-Tekya M, Dean JA. Histological evaluation of electrosurgery and formocresol pulpotomy techniques in primary teeth in dogs [abstract]. J Clin Pediatr Dent 2001; 26: 81 - 85 .

24. Needleman H, Hoang Ch, Allred E, Hertzberg J, Katz R. Reports of pain by children undergoing rapid palatal expansion. Ped Dent 2000; 22: $221-226$.

25. Parkell Sensimatic 500SE [Instruction Manual]. p. 2 - 11 .

26. Programa Epi - Info 6.02, OMS.

27. Magnusson B. Therapeutic pulpotomies in primary molars with the formocresol technique. A clinical and histological follow - up. Acta Odontol Scand 1978; 36: 157 - 165. 